\title{
THERMAL ANALYSIS OF AN IMPINGING JET ON A PLATE WITH AND WITHOUT A POROUS LAYER
}

\author{
Marcelo J. S. de Lemos and Cleges Fischer \\ Departamento de Energia - IEME, Instituto Tecnológico de Aeronáutica-ITA, \\ São José dos Campos, São Paulo, Brazil
}

This work shows numerical results for a jet impinging against a flat plane covered with a layer of a porous material, which is maintained at a higher temperature than the incoming fluid. Parameters such as permeability and thickness of the porous layer and thermal conductivity ration are varied in order to analyze their effects on the local distribution of $\mathrm{Nu}$. The macroscopic equations for mass, momentum, and energy are obtained based on a volumeaverage concept. The numerical technique employed for discretizing the governing equations was the control volume method with a boundary-fitted nonorthogonal coordinate system. The SIMPLE algorithm was used to handle the pressure-velocity coupling. Results indicate that inclusion of a porous layer decreases the peak in $\mathrm{Nu}$ avoiding excessive heating or cooling at the stagnation point. Also found was that the integral heat flux from the wall is enhanced for certain range of values of layer thickness, porosity, and thermal conductivity ratio.

\section{INTRODUCTION}

Impinging jets are commonly used in industrial applications with the objective of obtaining effective heating, cooling, or drying processes. The main advantage of using impinging jets is the possibility to obtain highly localized mass and heat transfer rates due to thin boundary layers inside of the stagnation region. Applications of such systems include electronics cooling, and glass tempering, metals cooling, and drying of textiles products and paper.

Published results are mainly concentrated on impinging jets under high mass flow conditions which reaches an uncovered surface. Pioneering studies considering two-dimensional impinging jets with low Reynolds number, also onto uncovered flat walls, are presented in Gardon and Akfirat [1], who experimentally obtained local and averaged heat transfer coefficients. Sparrow and Wong [2] made use of the well-known heat and mass transfer analogy and took experimental data on local mass transfer for a two-dimensional impinging jet. Results were then converted to heat transfer using the mentioned technique. Chen et al. [3] experimentally and numerically analyzed mass and heat transfer induced by a two-dimensional laminar jet.

Received 20 June 2008; accepted 18 August 2008.

The authors are thankful to CNPq and FAPESP for their financial support during the preparation of this work.

Address correspondence to Marcelo J. S. de Lemos, Departamento de Energia - IEME, Instituto Tecnológico de Aeronáutica - ITA, 12228-900 São José dos Campos, São Paulo, Brazil. E-mail: delemos@ ita.br 


\begin{tabular}{|c|c|c|c|}
\hline \multicolumn{4}{|c|}{ NOMENCLATURE } \\
\hline $\begin{array}{l}A_{i} \\
B \\
c_{F} \\
\mathrm{Da} \\
H \\
h \\
K \\
k_{\mathrm{eff}} \\
k \\
L \\
\mathrm{Nu} \\
p \\
q_{w} \\
q_{w}^{\phi} \\
\langle p\rangle^{i} \\
\mathrm{R}\end{array}$ & $\begin{array}{l}\text { macroscopic interface area between the } \\
\text { porous region and the clear flow } \\
\text { jet width } \\
\text { Forchheimer coefficient } \\
\text { Darcy number, } \mathrm{Da}=K / H^{2} \\
\text { channel height } \\
\text { porous layer thickness, film coefficient } \\
\text { permeability } \\
\text { effective thermal conductivity } \\
\text { thermal conductivity } \\
\text { channel length } \\
\text { Nusselt number } \\
\text { thermodynamic pressure } \\
\text { integral wall heat flux } \\
\text { integral wall heat flux will porous layer } \\
\text { intrinsic (fluid) average of pressure } p \\
\text { total drag per unit volume }\end{array}$ & $\begin{array}{l}\text { Subs } \\
s, f \\
w \\
\phi \\
o\end{array}$ & $\begin{array}{l}\text { Reynolds number based on the channe } \\
\text { height, } \mathrm{Re}=\rho\left|\mathbf{u}_{D}\right| H / \mu \\
\text { source term } \\
\text { temperature } \\
\text { intrinsic (fluid) average of } \mathbf{u} \\
\text { Darcy velocity vector (volume average } \\
\text { over } \mathbf{u})=\phi\langle\mathbf{u}\rangle^{i} \\
\text { Cartesian coordinates } \\
\text { dynamic viscosity } \\
\text { density }\end{array}$ \\
\hline
\end{tabular}

Chiriac and Ortega [4] performed numerical simulations in steady and transitory regime for a two-dimensional jet impinging against a plate with constant temperature. In recent years, a number of research papers have covered a wide range of studies in porous media [5-18], including flows parallel to a layer of porous material [19], across permeable baffles [20, 21], and porous inserts [22]. Investigation on configurations concerning perpendicular jets into a porous core is much needed for optimization of heat sinks attached to solid surfaces. However, studies of porous medium under impinging jets are, unfortunately, very scarce in the literature. An example found were those given by the numerical simulations of Kim \& Kuznetsov [23], who investigated optimal characteristics of impinging jets into heat sinks. Other innovative applications of impinging jets, such as fiber hydroentalglement, can also be found in recent literature [24, 25].

As mentioned, for the specific case investigated here, i.e., an impinging jet over a covered plate, there are not many results in the open literature; one example being the work of Prakash et al. (2001a,b) [26, 27]. They obtained a flow visualization of turbulent jets impinging against a porous medium. Also, Fu and Huang [28] evaluated the thermal performance of different porous layers under an impinging jet, and Jeng and Tzeng [29] studied the hydrodynamic and thermal performance of a jet impinging on a metallic foam. Recently, Graminho and de Lemos [30] investigated the flow structure of a laminar jet impinging on a layer of porous material. In ref. [30] a laminar version of a mathematical model described in detail by de Lemos [31], who worked on a macroscopic two-equation model for turbulent flow in porous media, was applied. Previously, Rocamora and de Lemos [32] added thermal modeling for the treatment of a permeable medium, and later, the model described in ref. [31] was expanded to handle buoyant flows [33, 34] and hybrid media consisting of both porous and clear regions [35].

The objective of the present contribution is to extend the isothermal investigation of Graminho and de Lemos [30] to a heat transfer analysis in order 
to evaluate under which circumstances the addition of a porous layer made of thermal conducting material can enhance the overall heat transferred from a flat surface under an impinging jet. By increasing the contact area between the coolant and the surface, larger amounts of heat can be extracted subjected to the same temperature difference. For example, such heat-sink devices are commonly used in the microcomputer industry to enhance the extraction of heat from ever-smaller microprocessors. Accordingly, porous metal foams conveniently installed over hot surfaces can enhance heating/cooling processes due to their high volumetric surface area (interfacial area per unit volume). Here, laminar flow is calculated based on the methodology given in refs. [31, 36].

\section{PROBLEM DESCRIPTION}

The cases here considered are detailed in Figure 1a. A laminar jet with uniform velocity $v_{o}$ and constant temperature $T_{o}$ enters through a gap into a channel with

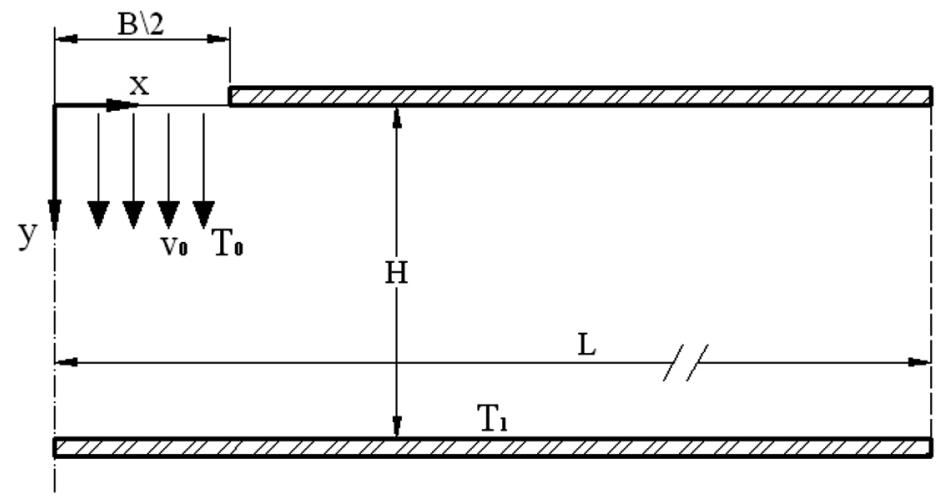

(a)

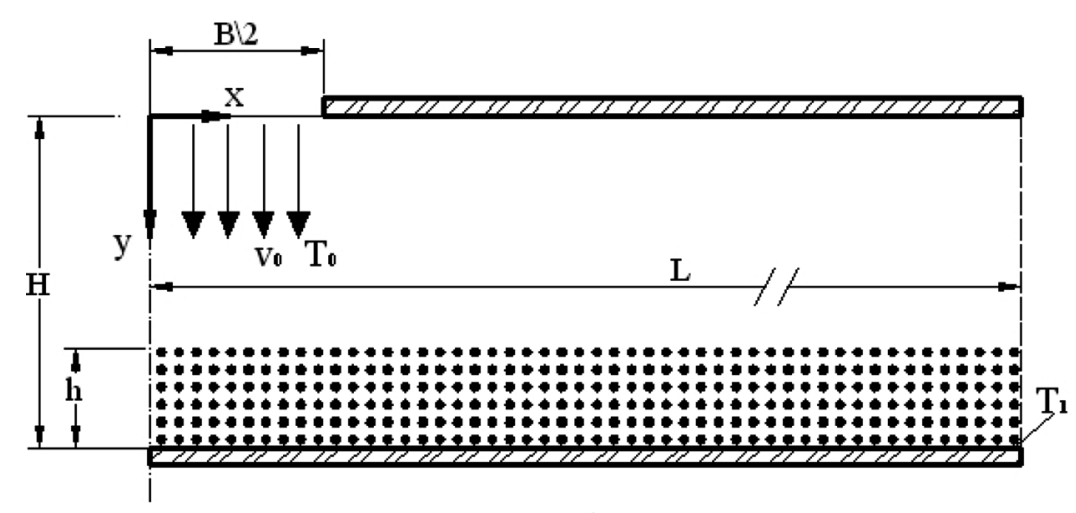

(b)

Figure 1. Cases investigated: (a) Confined impinging jet on a flat plate - clear medium case; and (b) confined impinging jet on a plate covered with a layer of porous material - porous case. 
height $H$ and length $2 L$. Fluid impinges normally against the bottom plate yielding a two-dimensional confined impinging jet configurarion. The width of the inlet nozzle is $B$ and the bottom plate temperature, $T_{1}$, is maintained constant and $10 \mathrm{~K}$ above the temperature of the incoming jet, $T_{o}$. In a different configuration, the bottom surface is covered with a porous layer of height $h$ (Figure $1 b$ ). In both cases, the flow is assumed to be two-dimensional, laminar, incompressible, and steady. Also, the porous medium is taken to be homogeneous, rigid, and inert. Fluid properties are constant and gravity effects are neglegted.

The boundary conditions for the problem are: 1) constant velocity and temperature profiles of the entering jet; 2) no slip condition on the walls; 3) symmetry condition in $x=0$; and 4) fully developed flow at channel exit $(x=L)$. At the bottom plate $(y=H)$, constant temperature condition is assumed whereas along the upper wall, for $B / 2<x \leq L$, null heat flux condition prevails.

\section{MATHEMATICAL MODELING AND NUMERICS}

The mathematical model employed here is described in Graminho and de Lemos [30] and now includes the energy equation for heat transfer calculations. Therein, details can be found. As most of the theoretical development is readily available in the open literature, the governing equations will just be presented and details about their derivations can be obtained in the referenced articles. Essentially, local instantaneous equations are volume-averaged using appropriate mathematical tools [37, 38]. Here, only laminar flow is considered since the Reynolds number based on the incoming jet velocity is less than 1000. Due to the expansion of the flow when entering the channel, velocities everywhere will always be lower than that of the incoming jet. For this reason, it is assumed here that the flow remains laminar in both the fluid layer and within the porous substrate. Accordingly, for laminar flow, the equations read as follows.

Macroscopic continuity equation

$$
\nabla \cdot \mathbf{u}_{D}=0
$$

where $\mathbf{u}_{D}$ is the average surface velocity (also known as Darcy velocity). In Eq. (1) the Dupuit-Forchheimer relationship, $\mathbf{u}_{D}=\phi\langle\mathbf{u}\rangle^{i}$, has been used, where $\phi$ is the porous medium porosity and $\langle\mathbf{u}\rangle^{i}$ identifies the intrinsic (fluid phase) average of the local velocity vector $\mathbf{u}$ [37].

Macroscopic momentum equation

$$
\rho \nabla \cdot \frac{\mathbf{u}_{D} \mathbf{u}_{D}}{\phi}=-\nabla \phi\langle p\rangle^{i}+\mu \nabla^{2} \mathbf{u}_{D}-\left[\frac{\mu \phi}{K} \mathbf{u}_{D}+\frac{c_{F} \phi \rho}{\sqrt{K}}\left|\mathbf{u}_{D}\right| \mathbf{u}_{D}\right]
$$

where the last two terms in Eq. (2) represent the Darcy and the Forchheimer terms, respectively. The symbol $K$ is the porous medium permeability, $c_{F}=0.55$ is the form drag coefficient (Forchheimer coefficient), $\langle p\rangle^{i}$ is the intrinsic pressure of the fluid, $\rho$ is the fluid density, and $\mu$ represents the fluid viscosity.

Macroscopic energy equation 
The macroscopic energy equation for laminar flow in porous medium, using the local thermal equilibrium hypothesis (LTE), has been given in detail in the work of Rocamora and de Lemos [32] as follows.

$$
\left(\rho c_{p}\right)_{f} \nabla \cdot\left(\mathbf{u}_{D}\langle T\rangle^{i}\right)=\nabla \cdot\left\{\mathbf{K}_{\mathrm{eff}} \cdot \nabla\langle T\rangle^{i}\right\}
$$

where $\langle T\rangle^{i}$ and $\mathbf{K}_{\text {eff }}$ are the average temperature (fluid and solid) and the effective conductivity tensors, respectively. The effective conductive tensor, $\mathbf{K}_{\mathrm{eff}}$, accounts for all mechanisms contributing to the energy budget, i.e., for laminar flow those mechanisms are effective conduction (fluid and solid), tortuosity, and thermal dispersion due to spatial deviations to temporal fluctuations and spatial deviations. $\mathbf{K}_{\text {eff }}$ is then given by

$$
\mathbf{K}_{\mathrm{eff}}=\left\lfloor\phi k_{f}+(1-\phi) k_{s}\right\rfloor \mathbf{I}+\mathbf{K}_{\mathrm{tor}}+\mathbf{K}_{\mathrm{disp}}
$$

where $\mathbf{K}_{\text {tor }}$ and $\mathbf{K}_{\text {disp }}$ are defined such that [39, 40]

Tortuosity

$$
\left[\frac{1}{\Delta V} \int_{A_{i}} \mathbf{n}\left(k_{f} \bar{T}_{f}-k_{s} \bar{T}_{s}\right) d S\right]=\mathbf{K}_{\mathrm{tor}} \cdot \nabla\langle\bar{T}\rangle^{i}
$$

Thermal dispersion

$$
-\left(\rho c_{p}\right)_{f}\left(\phi\left\langle^{i} \mathbf{u}^{i} \bar{T}_{f}\right\rangle^{i}\right)=\mathbf{K}_{\mathrm{disp}} \cdot \nabla\langle\bar{T}\rangle^{i}
$$

A complete review of Eq. (3) is beyond the scope of the present text and details of their derivation can be found in the reference mentioned above and in ref. [39]. Here, it is sufficient to say that the mechanism of dispersion comes from decomposing in space the convection term, and then volume-averaging it, giving [40]

$$
\left(\rho c_{p}\right)_{f} \nabla \cdot\left(\phi\langle\mathbf{u} T\rangle^{i}\right)=\left(\rho c_{p}\right)_{f} \nabla \cdot\left\{\phi(\underbrace{\langle\mathbf{u}\rangle^{i}\left\langle T_{f}\right\rangle^{i}}_{\text {convection }}+\underbrace{\left\langle\dot{i}^{i} \overline{\mathbf{T}}_{f}\right\rangle^{i}}_{\text {dispersion }})\right\}
$$

The last two terms on the right-hand side of Eq. (7) could be given the following physical significance. 1) Convective heat flux based on macroscopic time mean velocity and temperature, which yields the left of Eq. (3) after using $\mathbf{u}_{D}=\phi\langle\mathbf{u}\rangle^{i}$; and 2 ) thermal dispersion associated with deviations of mean velocity and temperature, which was modeled via Eq. (6).

Equations (1)-(3) subject to interface and boundary conditions were discretized in a two-dimensional control volume involving both clear and porous mediums. The finite-volume method was used in the discretization and the SIMPLE algorithm [41] was applied to handle the pressure-velocity coupling. The discretized form of the two-dimensional conservation equation for a generic property $\varphi$ in permanent regime reads

$$
I_{e}+I_{w}+I_{n}+I_{s}=S_{\varphi}
$$


where $I_{e}, I_{w}, I_{n}$, and $I_{s}$ represent, respectively, the fluxes of $\varphi$ in the east, west, north, and south faces of the control volume and $S_{\varphi}$ its term source.

Standard source term linearization is accomplished by using

$$
S_{\varphi} \approx S_{\varphi}^{* *}\langle\varphi\rangle_{p}^{i}+S_{\varphi}^{*}
$$

Discretization in the $x$ direction gives

$$
\begin{gathered}
S^{* x}=\left(S_{e}^{* x}\right)_{P}-\left(S_{w}^{* x}\right)_{P}+\left(S_{n}^{* x}\right)_{P}-\left(S_{s}^{* x}\right)_{P}+S_{P}^{*} \\
S^{* * x}=S_{\phi}^{* *}
\end{gathered}
$$

where $S^{* x}$ is the diffusive part, treated here in an explicit form. The second term, $S^{* * x}$, entails the additional drag forces due to the porous matrix, the last two terms in Eq. (2), and are treated here explicitly (see [30-34] for details).

For the numerical simulations presented here, a grid of $40 \times 150(6,000)$ nodes was used, which was refined next to the walls. Code validation and grid independency studies were conducted for the empty channel case and results were compared with the literature (not shown here due to a lack of space). Further, all results have been simulated with the following geometric configurations and boundary conditions: uniform inlet velocity and temperature profile; inlet jet temperature, $T_{o}=300 \mathrm{~K}$, bottom plate temperature, $T_{1}=310 \mathrm{~K}$; ratio between nozzle-to-plate distance and nozzle width, $H / B=2$; nozzle width, $B=1 \times 10^{-2} \mathrm{~m}$ and channel length, $L=0.5 \mathrm{~m}$.

\section{RESULTS AND DISCUSSION}

Impinging jets present three distinct characteristic regions according to Incropera and DeWitt [42] (see also Fox and McDonald [43] for basic information on impinging jets). The entrance region is termed the free jet, where the flow conditions are unaffected by the impingement (target) surface and the jet velocity is almost constant and equal to its nominal value. The stagnation region is where the flow is influenced by the impingement surface. There, the jet is decelerated and almost all kinetic energy is transformed into a static pressure rise. Following the impact, the jet flow is redirected and accelerated again along the target surface. The horizontal flow cannot accclerate indefinitely and becomes a decelerating wall jet, which comprises a boundary layer. The above characteristics of the flow around the stagnation region results in a peak of the Nusselt number $[42,43]$.

\section{Clear Channel}

The first set of results is related to the configuration shown in Figure $1 a$, where no porous material is attached to the bottom wall. Figure $2 a$ shows the distribution of the local Nusselt number close to the stagnation region compared with the experimental data of Gardon and Akfirat [1] and numerical results of Chen et al. [3]. In Figure $2 a$, the Reynolds number is $\mathrm{Re}=450$, the inlet velocity 


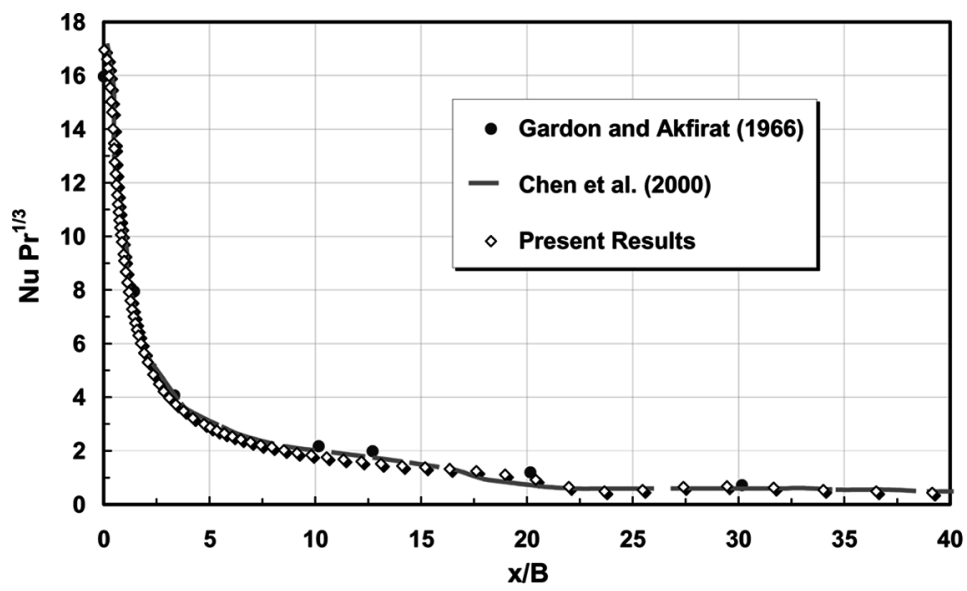

(a)

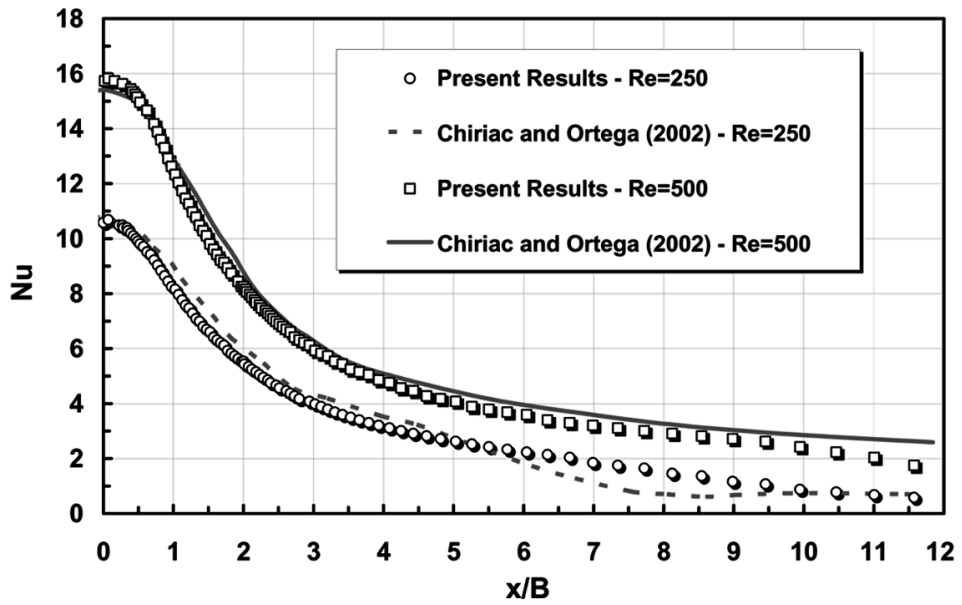

(b)

Figure 2. Validation for distribution of $\mathrm{Nu}$ along the lower plate for clear channel. (a) $\mathrm{Re}=450, H / B=4$ and $(b) H / B=5$.

profile at the jet inlet is fully developed, and the fluid flows in between parallel plates. The temperature profile is uniform at the bottom wall and the ratio between the nozzle-to-plate spacing and nozzle width is $H / B=4$. In order to make the comparison it is necessary to normalize the local Nusselt with $\operatorname{Pr}^{-1 / 3}$. Figure $2 b$ shows a comparison of the local Nusselt number at the lower plate with the numerical results of Chiriac and Ortega [4]. Inlet velocity and temperature profiles are uniform at the entrance slot and $H / B=5$. In addition, one can see that a reasonable agreement with the literature is obtained, for a Reynolds number spanning from 250 to 500. Overall, Figure 2 seems to show that results agree well with the values found in the literature, indicating the correctness of the code used as well as proper grid validation. 

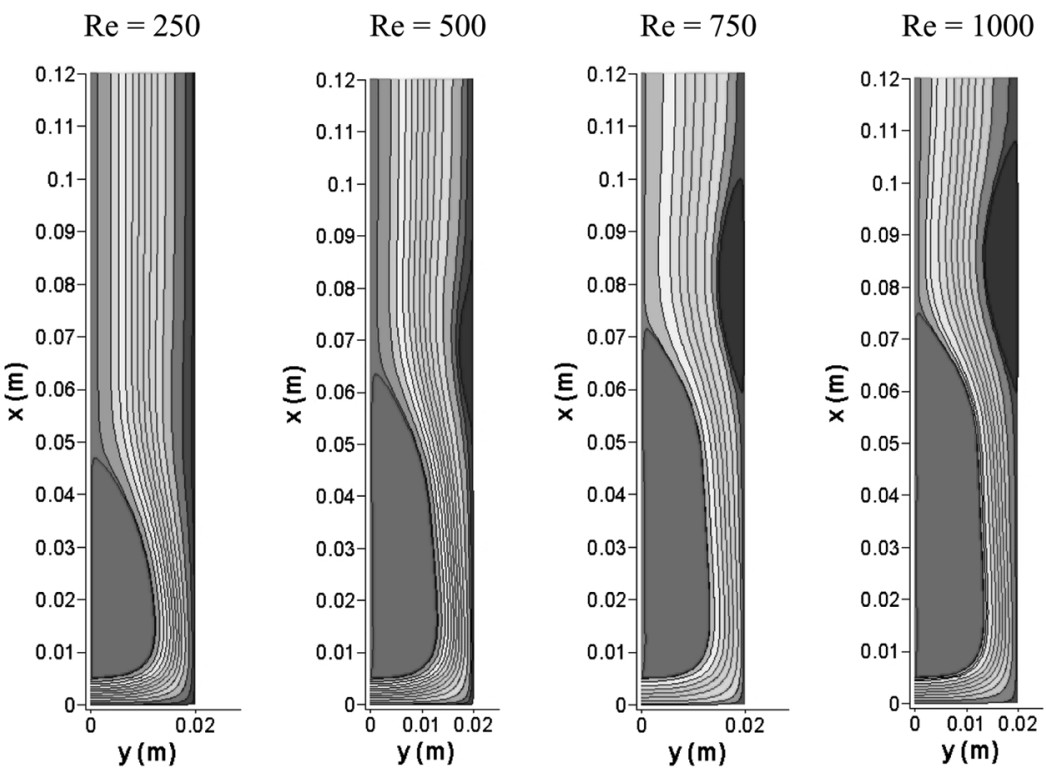

(a)
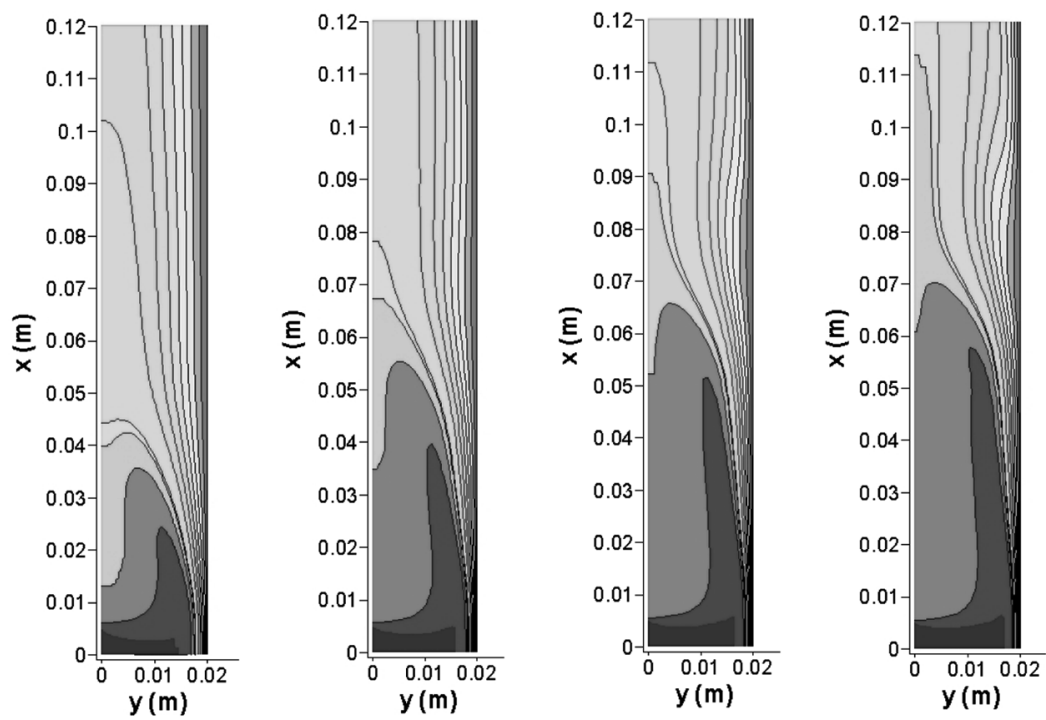

(b)

Figure 3. Results for uncovered lower wall (clear medium) and $H / B=2$. (a) streamlines and (b) temperature distribution.

Figure 3 shows streamlines and temperature fields for various Reynolds numbers for the condition of an empty channel (Figure 1a). Note that the figures are turned counterclockwise so that the jet entrance is at the bottom left corner of the drawings. Figure $3 a$ shows that a primary recirculation near the inlet jet region 
appears, caused by the sudden expansion of the jet when penetrating the stagnant fluid within the channel. With an increase in Reynolds number, a secondary recirculation bubble arises close to the bottom, further downstream the jet axis. In Figure $3 b$, the temperature mixing inside the recirculating regions is evident. Strong temperature gradients close to the bottom wall are also noted, which get even higher as Re increases and the thermal boundary layer thickness is reduced.

For a performance analysis of heat transfer due to the jet velocity variation, Figure 4 shows a graph with the local Nusselt number close to the target plate for various Reynolds numbers. The Nusselt along the bottom wall is calculated as

$$
\mathrm{Nu}=\frac{h H}{k_{\mathrm{eff}}}=\left(\frac{\partial\langle T\rangle^{v}}{\partial y}\right)_{y=H} \frac{H}{T_{1}-T_{0}} \quad \text { where } k_{\text {eff }}=\phi k_{f}+(1-\phi) k_{s}
$$

and $h$ is a film coefficient.

According to Figure 4, as the Reynolds number increases the Nusselt number peak at the stagnation region $(x=0)$ increases. In addition, it can be seen that a second Nusselt peak appears for a Reynolds number greater than $\mathrm{Re}=500$ and at about $x / B=10$. This second peak is related to the second recirculation region shown above in Figure $3 a$, and can be attributed to the engulfing motion around the secondary recirculation region which reduces the thickness of the thermal boundary layer [44].

\section{Channel with Porous Layer}

When a layer of porous material is added to the bottom of the channel, the resulting configuration is shown in Figure $1 b$. The material is assumed to be rigid,

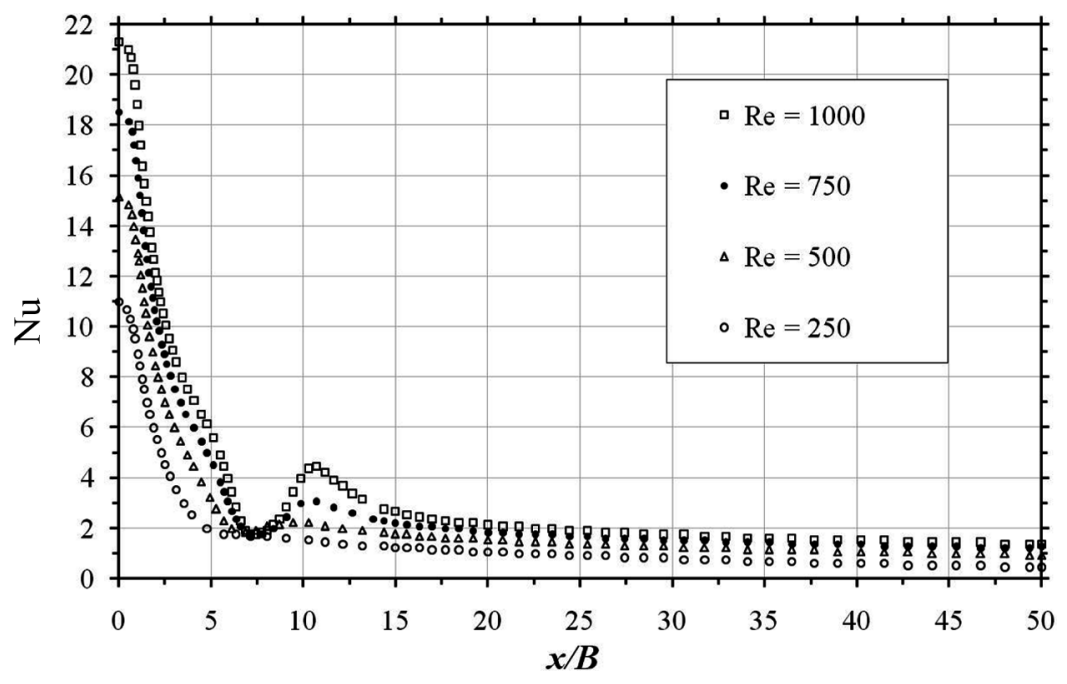

Figure 4. Local Nusselt distribution close to the inferior plate for clean medium and $H / B=2$. 
with porosity $\phi$, permeability $K\left(\mathrm{Da}=K / H^{2}\right)$, and nondimensional thickness $h / H$. Results below are obtained using distinct values for such three parameters.

Effect of porosity, $\phi$. Figure 5 shows the streamlines and temperature fields for a porous layer of thickness $h / H=0.5$. The Reynolds number is maintained constant and equal to 750 .
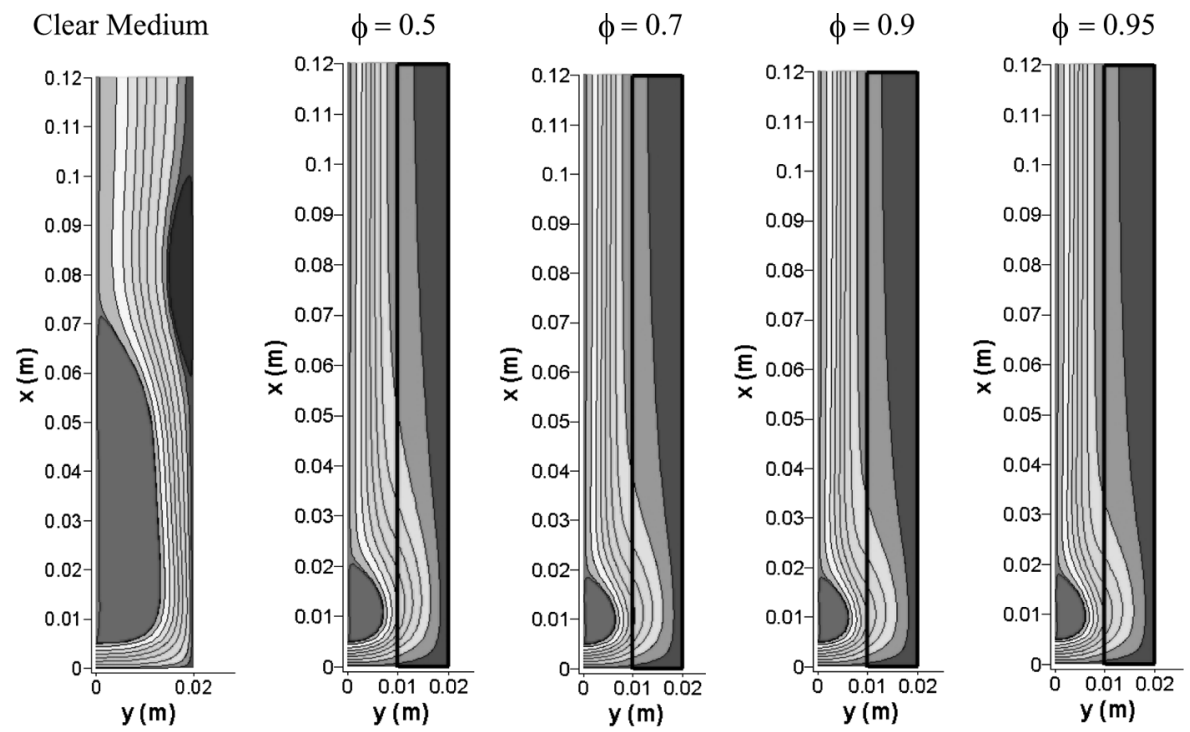

(a)
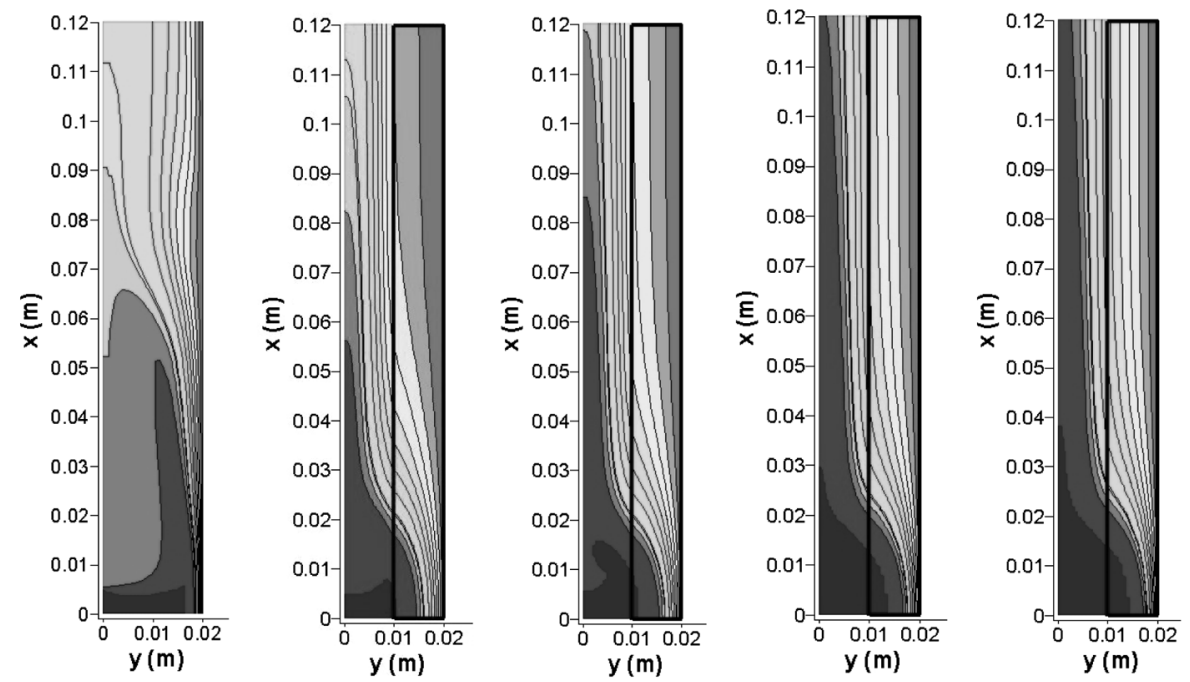

(b)

Figure 5. Effect of porosity for $\mathrm{Re}=750, H / B=2, \mathrm{Da}=8.28 \times 10^{-3}, h=0.5 H$, and $k_{s} / k_{f}=10$. (a) streamlines and (b) temperature field. 
Figure $5 a$ indicates that porosity variation does not strongly influence the flow behavior, as also confirmed by Graminho and de Lemos [30]. On the other hand, in Figure $5 b$ it is possible to note that as porosity increases, the temperature field is more quickly homogenized. Also, as porosity increases, the jet penetrates into the permeable material reaching the bottom plate more easily. Consequently, the thermal boundary layer is compressed to the wall at the stagnation region. A greater temperature gradient results at the wall, reflecting in an enhancement of heat transfer therein.

In order to further analyze the porosity influence on heat transfer, the distribution of the local Nusselt number is plotted as seen in Figure 6. Again, the configuration has the insertion of a porous layer of relative thickness $h=0.5 H$, the Reynolds number is maintained constant and equal to $\mathrm{Re}=750$, and the permeability is $K=3.31 \times 10^{-6} \mathrm{~m}^{2}$. Inspecting the figure, one can note that the insertion of a porous layer eliminates the second peak in $\mathrm{Nu}$ distribution, leaving only the Nusselt peak at the stagnation region. Further away form $x=0, \mathrm{Nu}$ decreases to a minimum value, which is kept constant thereafter. In addition, a porosity increase implies an increase in stagnation of the Nusselt number. Then, with the insertion of a porous layer the distribution of the Nusselt number becomes more homogeneous, which is an indication of a better heat transfer distribution along the plate.

Effect of channel blockage, $\boldsymbol{h} / \boldsymbol{H}$. A study of the influence of the porous layer thickness in the heat transfer is now presented. The streamlines and the field temperature for a simulation with various porous layer thicknesses, with $\mathrm{Re}=750$, $H / B=2, \mathrm{Da}=8.28 \times 10^{-3}\left(K=3.31 \times 10^{-6} m^{2}\right)$, and $\phi=0.90$ are presented in Figure 7.

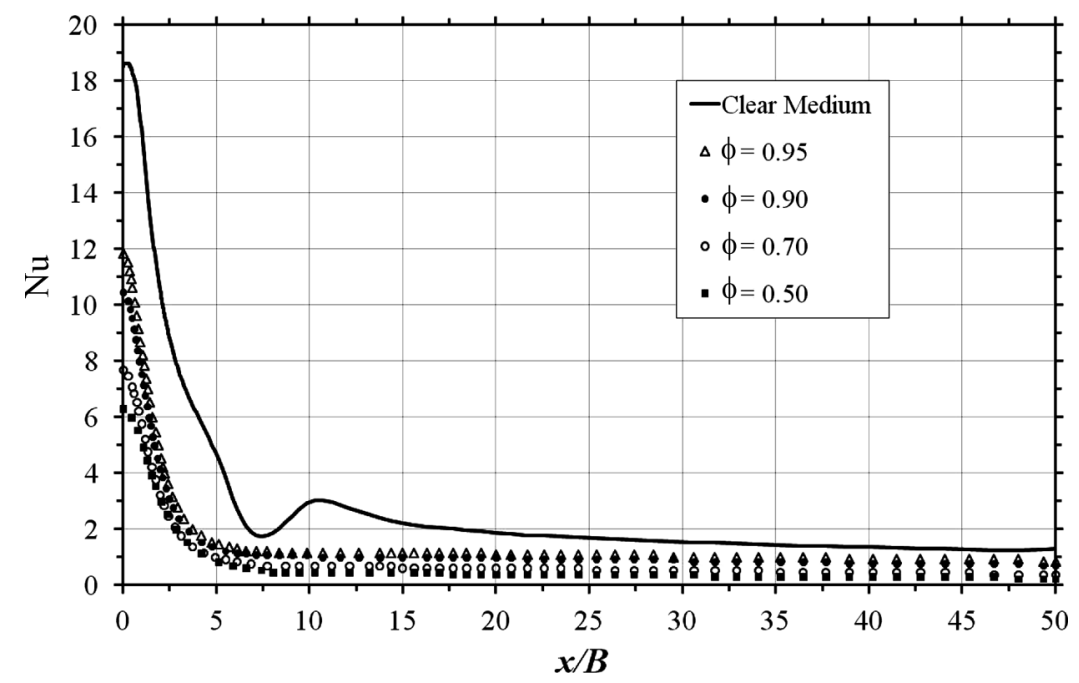

Figure 6. Local Nusselt distribution for various porosities with $\mathrm{Re}=750, H / B=2, \mathrm{Da}=8.28 \times 10^{-3}$, $k_{s} / k_{f}=10$, and $h / H=0.5$. 

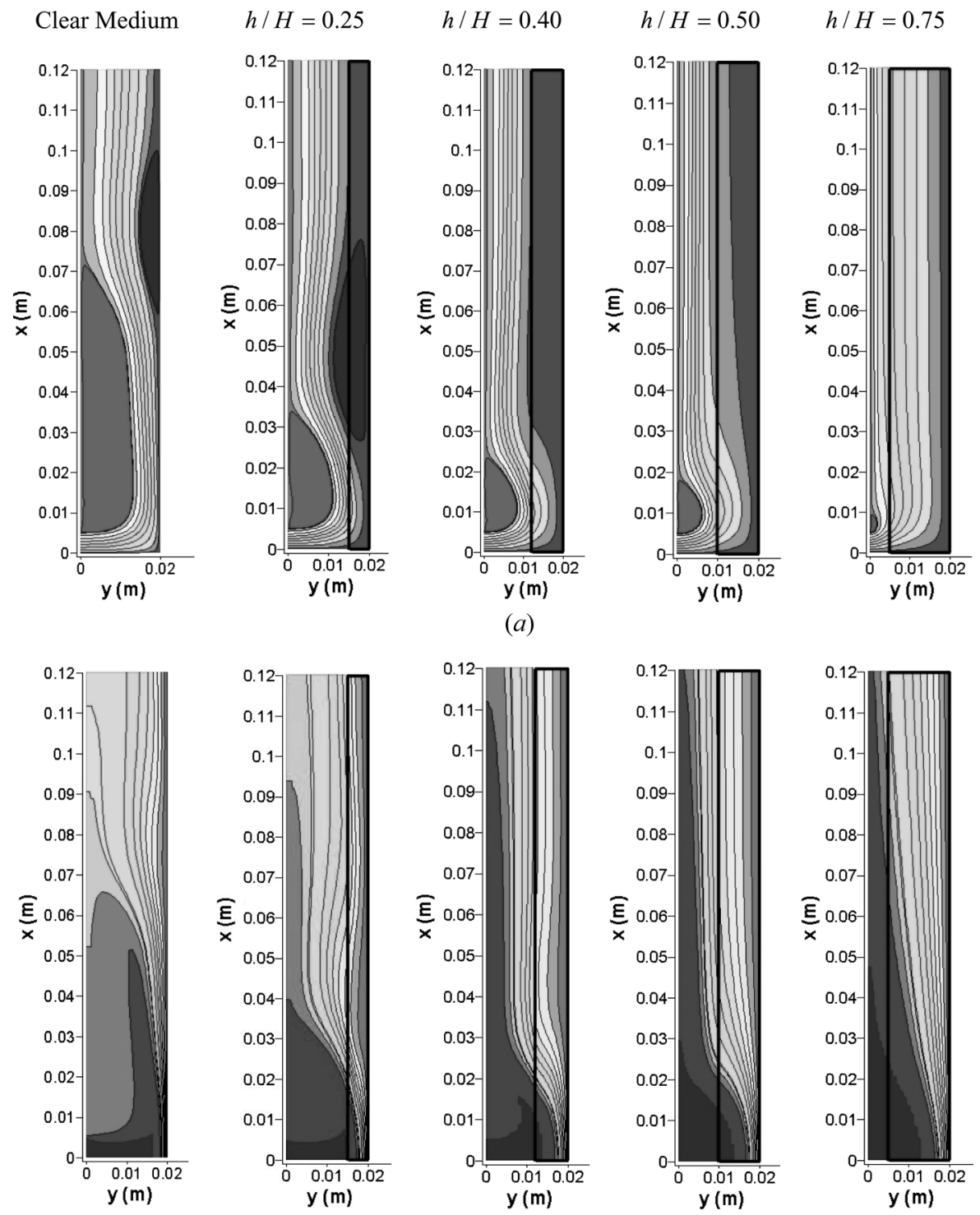

(b)

Figure 7. Effect of porous layer thicknesses for $\mathrm{Re}=750, H / B=2, \mathrm{Da}=8.28 \times 10^{-3}, \phi=0.9$, and $k_{s} / k_{f}=10 .(a)$ streamlines and $(b)$ temperature field.

Figure $7 a$ shows that porous layer thickness strongly influences the flow behavior. For $h=0.25 H$, the primary vortex diminishes and the second vortex has its size increased. But increasing $h$ from $h=0.25 H$, the secondary vortex disappears and the primary diminishes its size. The temperature field also is influenced by the porous layer thickness variation, as shown in Figure $7 b$. With thickness until $h=0.50 H$, the direction of the isothermals vary more strongly, mainly in the 


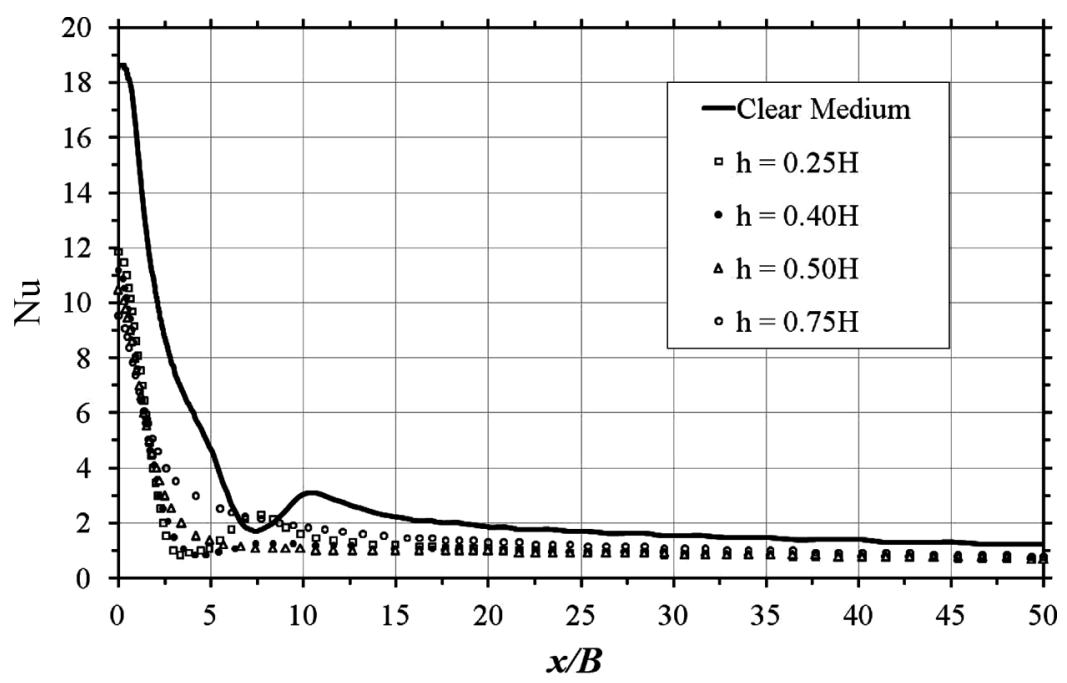

Figure 8. Local Nusselt distribution for various porous layer thicknesses with $\operatorname{Re}=750, H / B=2$, $\mathrm{Da}=8.28 \times 10^{-3}, k_{s} / k_{f}=10$, and $\phi=0.9$.

stagnation region, but it does not happen to the thickness $h=0.75 H$, where the isothermals lightly vary throughout the temperature field.

To complete the analysis, the local Nusselt number for various thickness of porous layer is presented in Figure 8. The stagnation of the Nusselt peak diminishes with the insertion of the porous layer, and for thicknesses inferior to $h=0.40 \mathrm{H}$ the second Nusselt peak continues, as shown in Figure 8. The presence of the second Nusselt peak is connected with the secondary vortices that appear in Figure $7 a$. Also, it can be seen that the variation of the thickness of porous layer does not influence the value of stagnation of the Nusselt peak as strongly as the variation of porosity, as can be concluded by comparing Figures 6 and 8. The main influence of thickness of the porous layer is the change in the curve shape of Nusselt distribution once the Nusselt peak value and the minimum constant Nusselt value are similar for all thickness.

Effect of Darcy number, Da. The effect of Darcy number $\mathrm{Da}=K / H^{2}$ is presented in Figure 9. Streamlines displayed in Figure $9 a$ indicate that, as Da decreases, fluid is pushed to flow above the porous slayer in the clear passage of size $0.5 \mathrm{H}$. Also noted is the reduction and elongation of the recirculating bubble, no longer penetrating the porous substrate as the permeability is reduced. Corresponding temperature distributions are plotted in Figure $9 b$. For low Da cases, the penetrating flow is sufficiently strong to deform the temperature and form a thin boundary layer at the jet impinging region. As Da is reduced, the thermal boundary layer becomes thicker at the jet axis location and heat is transported through the porous material nearly by conduction. For $\mathrm{Da}=4.50 \times 10^{-5}$, the porous substrate behaves like a solid layer and a boundary layer forms at the macroscopic interface between the two media. Nusselt numbers distribution in Figure 10 are plotted for corresponding Da. Reduction of the layer permeability thickens the thermal boundary layer at the 

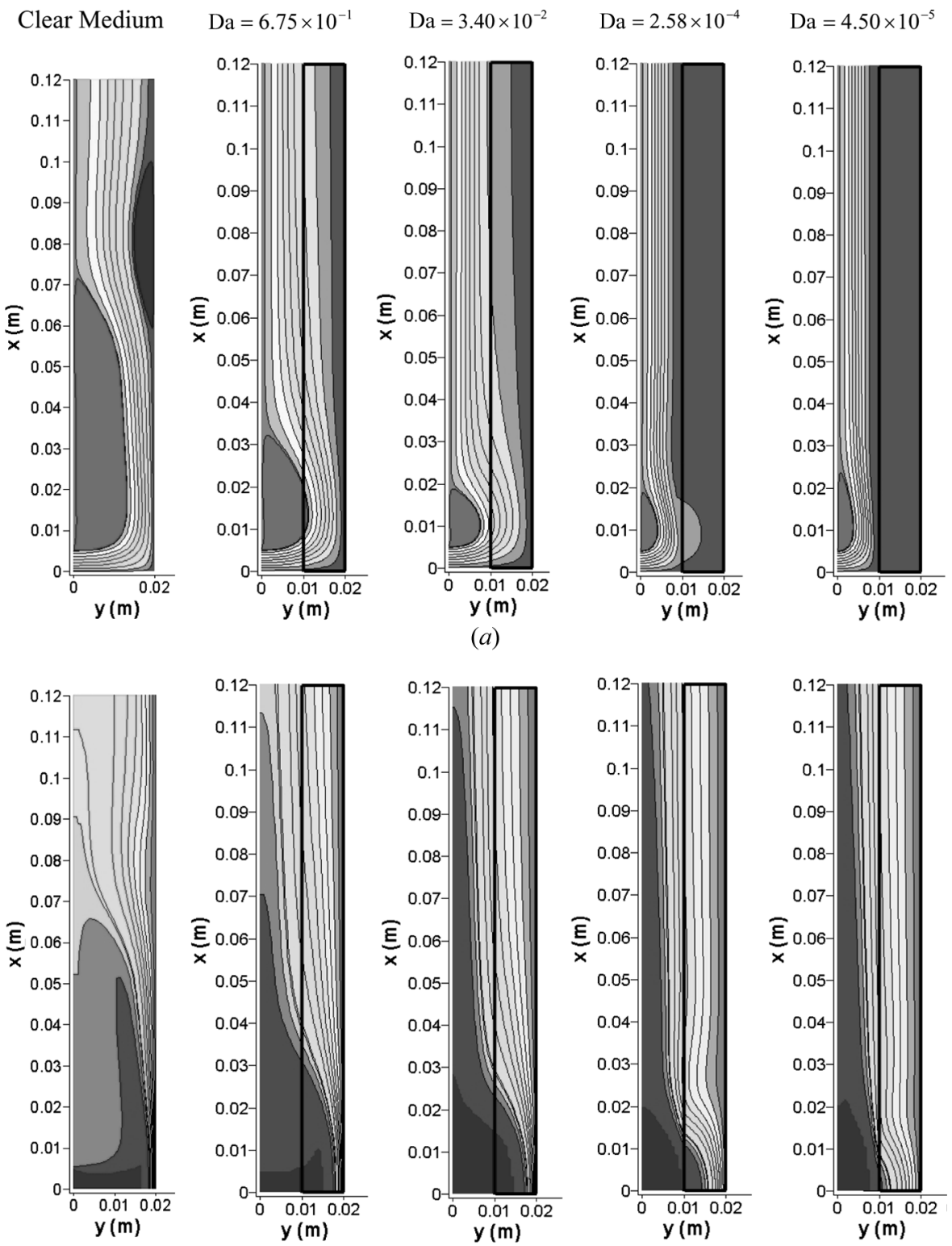

(b)

Figure 9. Effect of $\mathrm{Da}$ for $\mathrm{Re}=750, H / B=2, h / H=0.5, \phi=0.9$, and $k_{s} / k_{f}=10$. (a) streamlines and (b) temperature field.

stagnation point, leading to a reduction of $\mathrm{Nu}$ therein. Homogenization of temperature gradient along $x / B$ also eliminates the peak in $\mathrm{Nu}$. Also, for Da less than $3.40 \times 10^{-2}$, no substantial variation on $\mathrm{Nu}$ is detected, possibly indicating an optimal value for $\mathrm{Da}$ if the objective of such system is to control heat transfer from the wall. 


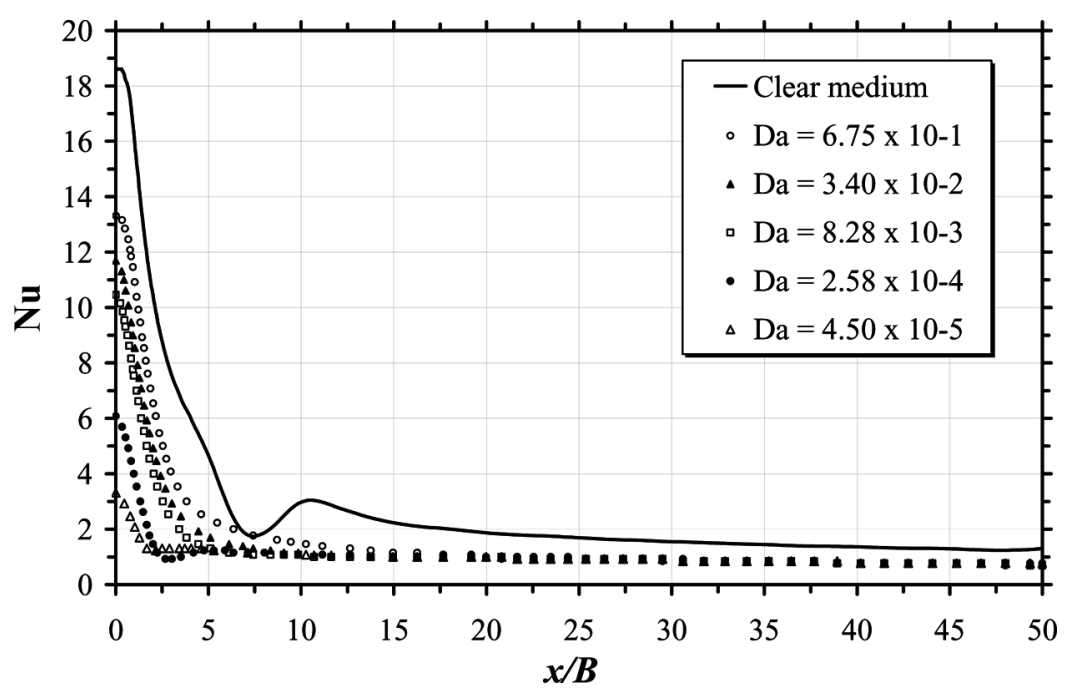

Figure 10. Local Nusselt distribution for various permeabilities with $\operatorname{Re}=750, H / B=2, h / H=0.5$, $\phi=0.9$, and $k_{s} / k_{f}=10$.

\section{Integral Wall Heat Flux}

Another important parameter to evaluate the effectiveness in using porous layer is to calculate the integrated heat transferred from the bottom wall. Such overall heat transferred from the lower wall to the flowing fluid can be calculated for both configurations, presented in Figure 1, as

$$
q_{w}=\frac{1}{L} \int_{o}^{L} q_{w x}(x) d x \quad q_{w x}=-\left.k_{\mathrm{eff}} \frac{\partial\langle T\rangle^{i}}{\partial y}\right|_{y=H}
$$

For the cases where the a porous layer is considered, the wall hat flux is given a superscript $\phi$ on the form $q_{w}^{\phi}$. The ratio $q_{w}^{\phi} / q_{w}$ can then be seen as a measure of the effectiveness of using a porous layer for enhancing or damping the amount of heat transferred through the wall.

Figure 11 compares the ratio $q_{w}^{\phi} / q_{w}$ for several porosities and porous layer height $h / H$, with $k_{s} / k_{f}=10$. The figure suggests that for cases where there is a more solid material per unit volume, or for low $\phi$, there is a net gain when using a porous substrate covering the cooled wall $\left(q_{w}^{\phi} / q_{w}>1\right)$. This is more apparent as the layer gets thicker (Figure 11c). On the other hand, for very high porosities and relatively high $\mathrm{Re}$, disruption of the thermal boundary layer close to the wall, due to the presence of a porous material, damps the overall heat transferred from the wall.

Figure 12 further shows variations for the integrated wall heat flux ratio with $h / H$ for two values of porosity $\phi$. For a medium with low porosity or more solid material (Figure 12a), the use of a porous substrate is always beneficial, regardless of the layer thickness. On the other hand, light material may enhance heat transfer only for thicker layer cases, whose limiting thickness increases with increasing Re, 


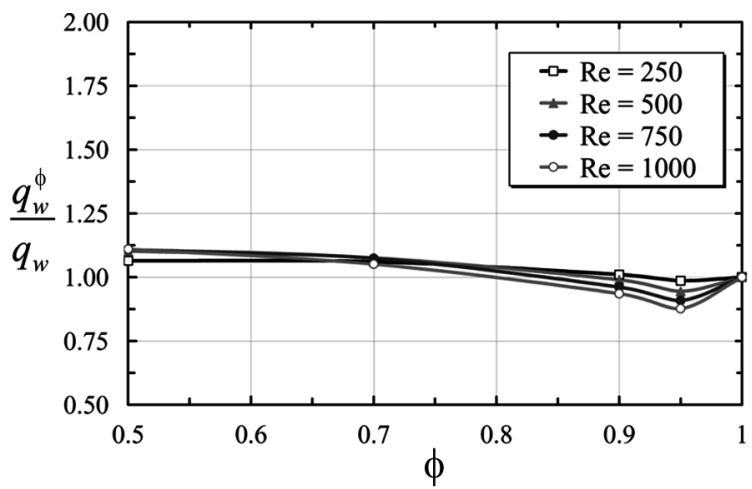

(a)

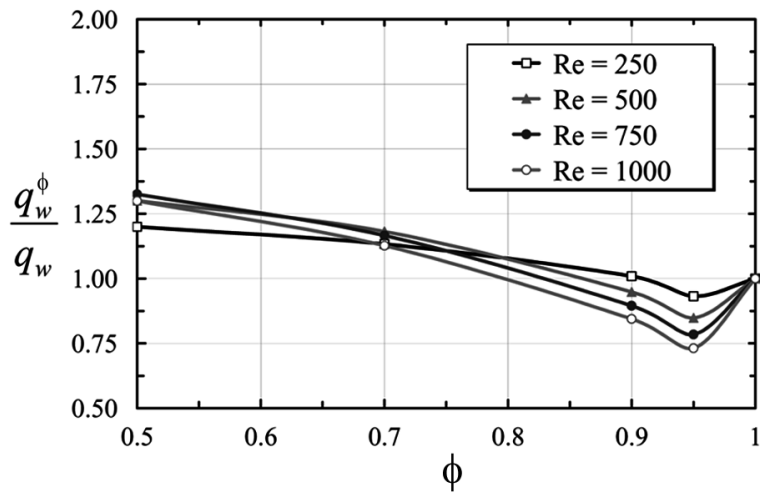

(b)

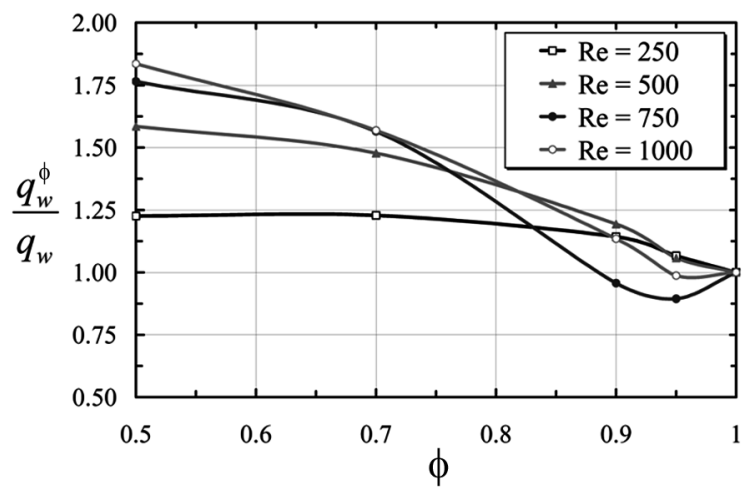

(c)

Figure 11. Integral heat flux ratio at the lower wall for various Reynolds and porosities with $H / B=2$, $\mathrm{Da}=8.28 \times 10^{-3}$, and $k_{s} / k_{f}=10 .(a) h / H=0.25,(b) h / H=0.5$, and $(c) h / H=0.75$.

i.e., for $\mathrm{Re}=250$, Figure $12 b$ indicates a gain in $q_{w}$ for $h / H \geq 0.5$, whereas for $\mathrm{Re}=1000$ the layer needs to have $h / H \geq 0.63$ for getting $q_{w}^{\phi} / q_{w}>1$.

Finally, Figure 13 compiles results for $q_{w}^{\phi} / q_{w}$ when the conductivity ratio $k_{s} / k_{f}$ is varied. Here, it is important to emphasize that results in the figure were obtained 


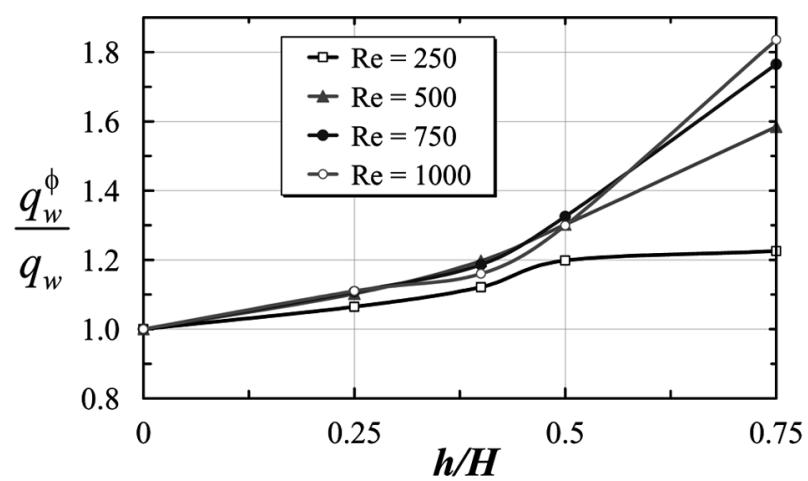

(a)

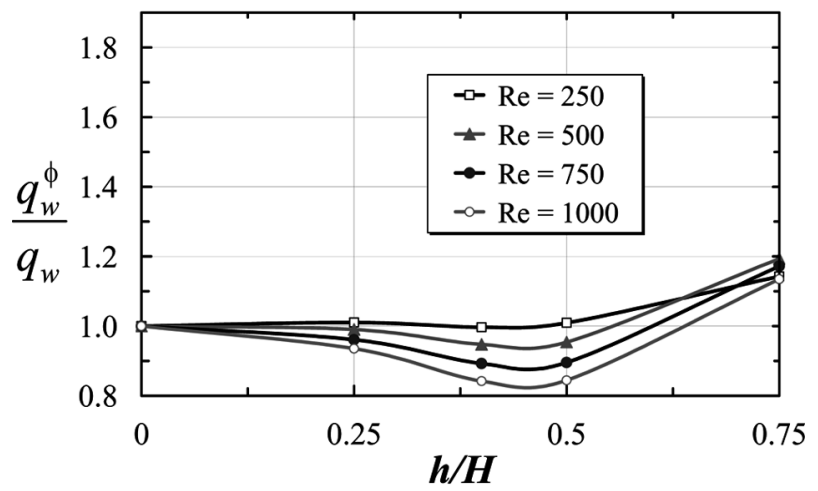

(b)

Figure 12. Integral heat flux ratio at the lower wall for various Reynolds and porous layer thicknesses with $H / B=2, \mathrm{Da}=8.28 \times 10^{-3}$, and $k_{s} / k_{f}=10 .(a) \phi=0.5$ and $(b) \phi=0.9$.

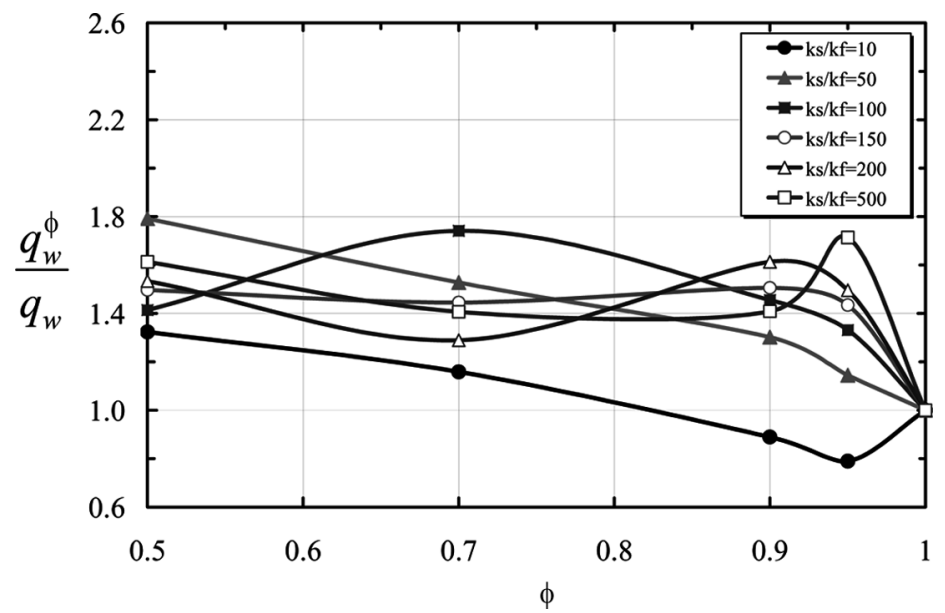

Figure 13. Integral heat flux ratio at the lower wall for various ratios $k_{s} / k_{f}$ for $\operatorname{Re}=750, H / B=2$, and $\mathrm{Da}=8.28 \times 10^{-3}$ 
with the local thermal equilibrium (LTE) assumption $\left(\langle T\rangle^{i}=\left\langle T_{f}\right\rangle^{i}=\left\langle T_{s}\right\rangle^{i}\right.$ ). Such hypotheses might not be valid when the conductivity of the two media differs from each other by a large amount. As such, results herein should be seen with caution and as a first approximation of a more elaborate calculation using the so called two-energy equation model.

One can note in Figure 13 that increasing the conductivity ratio past $k_{s} / k_{f}>10$, it is always possible to enhance the heat extracted from the bottom wall. Also, for $k_{s} / k_{f}>100$, computations seem to be scattered and no coherent pattern is detected when $k_{s} / k_{f}$ is varied. This seemingly erratic behavior of $q_{w}^{\phi} / q_{w} \times k_{s} / k_{f}$ might be a consequence of the unsuitability of the LTE model for cases where the thermal conductivity of the solid is substantially higher than $k_{f}$. In those cases, distinct energy equations for each phase might be a more realistic model to use.

\section{CONCLUSIONS}

The presence of a porous layer on the plate eliminates the second peak in $\mathrm{Nu}$ and allows for controlling heat transfer from the wall. It was observed that porosity strongly influences the stagnation Nusselt value, while the porous layer thickness affects more intensely the distribution of $\mathrm{Nu}$ along the plate. Low porosity material tends to yield a better heat absorption when compared to media that are more permeable. Increasing the thermal conductivity ratio is always beneficial to heat transfer enhancement form the hot wall. Ultimately, results in this work might be useful to engineers designing systems that make use of impinging jets over thermally conducting porous materials, which possesses a large interfacial air-to-solid contact area and are used to advantage for cooling or heating purposes.

\section{REFERENCES}

1. R. Gardon and J. C. Akfirat, Heat Transfer Characteristics of Impinging TwoDimensional Air Jets, J. Heat Transfer, vol. 101, pp. 101-108, 1966.

2. E. M. Sparrow and T. C. Wong, Impinging Transfer Coefficients due to Initially Laminar Slot Jets, Inter. J. Heat and Mass Transfer, vol. 18, pp. 597-605, 1975.

3. M. Chen, R. Chalupa, A. C. West, and V. Modi, High Schmidt Mass Transfer in a Laminar Impinging Slot Jet, Inter. J. Heat and Mass Transfer, vol. 43, pp. 3907-3915, 2000.

4. V. A. Chiriac and A. Ortega, A Numerical Study of the Unsteady Flow and Heat Transfer in a Transitional Confined Slot Jet Impinging on an Isothermal Surface, Inter. J. Heat and Mass Transfer, vol. 45, pp. 1237-1248, 2002.

5. Y. Zhang, X. F. Peng, and I. Conte, Heat and Mass Transfer with Condensation in Non-Saturated Porous Media, Numer. Heat Transfer A, vol. 52, pp. 1081-1100, 2007.

6. M. E. Taskin, A. G. Dixon, and E. H. Stitt, CFD Study of Fluid Flow and Heat Transfer in a Fixed Bed of Cylinders, Numer. Heat Transfer A, vol. 52, no. 3, pp. 203-218, 2007.

7. T. Basak, S. Roy, and H. S. Takhar, Effects of Nonuniformly Heated Wall(s) on a Natural-Convection Flow in a Square Cavity Filled with a Porous Medium, Numer. Heat Transfer A, vol. 51, no. 10, pp. 959-978, 2007.

8. J. Eriksson, S. Ormarsson, and H. Petersson, Finite-Element Analysis of Coupled Nonlinear Heat and Moisture Transfer in Wood, Numer. Heat Transfer A, vol. 50, no. 9, pp. 851-864, 2006. 
9. V. Bubnovich, L. Henriquez, and N. Gnesdilov, Numerical Study of the Effect of the Diameter of Alumina Balls on Flame Stabilization in a Porous-Medium Burner, Numer. Heat Transfer A, vol. 52, no. 3, pp. 275-295, 2007.

10. X. B. Chen, P. Yu, S. H. Winoto, and H. T. Low, Free Convection in a Porous Wavy Cavity Based on the Darcy-Brinkman-Forchheimer Extended Model, Numer. Heat Transfer A, vol. 52, no. 4, pp. 377-397, 2007.

11. N. Yucel and R. T. Guven, Forced-Convection Cooling Enhancement of Heated Elements in a Parallel-Plate Channels using Porous Inserts, Numer. Heat Transfer A, vol. 51, no. 3, pp. 293-312, 2007.

12. L. Betchen, A. G. Straatman, and B. E. Thompson, A Nonequilibrium Finite-Volume Model for Conjugate Fluid/Porous/Solid Domains, Numer. Heat Transfer A, vol. 49, no. 6, pp. 543-565, 2006.

13. Y. W. Zhang, Nonequilibrium Modeling of Heat Transfer in a Gas-Saturated Powder Layer Subject to a Short-Pulsed Heat Source, Numer. Heat Transfer A, vol. 50, no. 6, pp. 509-524, 2006.

14. C. R. Ruivo, J. J. Costa, and A. R. Figueiredo, Analysis of Simplifying Assumptions for the Numerical Modeling of the Heat and Mass Transfer in a Porous Desiccant Medium, Numer. Heat Transfer A, vol. 49, no. 9, pp. 851-872, 2006.

15. F. Moukalled and Y. Saleh, Heat and Mass Transfer in Moist Soil, Part I. Formulation and Testing, Numer. Heat Transfer A, vol. 49, no. 5, pp.467-486, 2006.

16. X. H. Wang, M. Quintard, and G. Darche, Adaptive Mesh Refinement for OneDimensional Three-Phase Flow with Phase Change in Porous Media, Numer. Heat Transfer B, vol. 50, no. 3, pp. 231-268, 2006.

17. X. H. Wang, M. Quintard, and G. Darche, Adaptive Mesh Refinement for OneDimensional Three-Phase Flow with Phase Change in Porous Media, Numer. Heat Transfer $A$, vol. 50, no. 4, pp. 315-352, 2006.

18. A. Mansour, A. Amahmid, M. Hasnaoui, and M. Bourich, Multiplicity of Solutions Induced by Thermosolutal Convection in a Square Porous Cavity Heated from Below and Submitted to Horizontal Concentration Gradient in the Presence of Soret Effect, Numer. Heat Transfer A, vol. 49, no. 1, pp. 69-94, 2006.

19. A. V. Kuznetsov, L. Cheng, and M. Xiong, Effects of Thermal Dispersion and Turbulence in Forced Convection in a Composite Parallel-Plate Channel: Investigation of Constant Wall Heat Flux and Constant Wall Temperature Cases, Numer. Heat Transfer A, vol. 42, no. 4, pp. 365-383, 2002.

20. B. M. D. Miranda and N. K. Anand, Convective Heat Transfer in a Channel with Porous Baffles, Numer. Heat Transfer A, vol. 46, no. 5, pp. 425-452, 2004.

21. N. B. Santos and M. J. S. de Lemos, Flow and Heat Transfer in a Parallel-Plate Channel with Porous and Solid Baffles, Numer. Heat Transfer A, vol. 49, no. 5, pp. 471-494, 2006.

22. M. Assato, M. H. J. Pedras, and M. J. S. de Lemos, Numerical Solution of Turbulent Channel Flow Past a Backward-Facing-Step with a Porous Insert Using Linear and Non-Linear k-E Models, J. Porous Media, vol. 8, no. 1, pp. 13-29, 2005.

23. S. Y. Kim and A. V. Kuznetsov, Optimization of Pin-Fin Heat Sinks using Anisotropic Local Thermal Nonequilibrium Porous Model in a Jet Impinging Channel, Numer. Heat Transfer A, vol. 44, no. 8, pp. 771-787, 2003.

24. P. Xiang, A. V. Kunetsov, and A. M. Seyam, A Porous Medium Model of the Hydroentanglement Process, J. Porous Media, vol. 11, pp. 35-49, 2008.

25. P. Xiang and A. V. Kunetsov, Simulation of Shape Dynamics of a Long Flexible Fiber in a Turbulent Flow in the Hydroentanglement Process, Inter. Comm. Heat and Mass Transfer, vol. 35, pp. 529-534, 2008.

26. M. Prakash, F. O. Turan, Y. Li, J. Manhoney, and G. R. Thorpe, Impinging Round Jet Studies in a Cilindrical Enclosure with and without a Porous Layer: Part I. 
Flow Visualizations and Simulations, Chem. Eng. Sci., vol. 56, pp. 3855-3878, 2001a.

27. M. Prakash, F. O. Turan, Y. Li, J. Manhoney, and G. R. Thorpe, Impinging Round Jet Studies in a Cilindrical Enclosure with and without a Porous Layer: Part II. DLV Measurements and Simulations, Chem. Eng. Sci., vol.56, pp. 38793892, $2001 \mathrm{~b}$.

28. W.-S. Fu and H.-C. Huang, Thermal Performance of Different Shape Porous Blocks under an Impinging Jet, Inter. J. Heat and Mass Transfer, vol. 40, no. 10, pp. 22612272, 1997.

29. T.-Z. Jeng and S.-C. Tzeng, Numerical Study of Confined Slot Jet Impinging on Porous Metallic Foam Heat Sink, Inter. J. Heat and Transfer, vol. 48, pp. 4685-4694, 2005.

30. D. R. Graminho and M. J. S. de Lemos, Laminar Confined Impinging Jet into a Porous Layer, Numer. Heat Transfer, vol. 54, no. 2, pp. 151-177, 2008.

31. M. J. S. de Lemos, Turbulence in Porous Media: Modeling and Applications, Elsevier, Amsterdam, 2006.

32. F. D. Rocamora Jr. and M. J. S. de Lemos, Analysis of Convective Heat Transfer of Turbulent Flow in Saturated Porous Media, Int. Comm. Heat and Mass Transfer, vol. 27 , no. 6 , pp. $825-834,2000$.

33. M. J. S. de Lemos and E. J. Braga, Modeling of Turbulent Natural Convection in Saturated Rigid Porous Media, Inter. Comm. Heat and Mass Transfer, vol. 30, no. 5, pp. 615-624, 2003.

34. E. J. Braga and M. J. S. de Lemos, Heat Transfer in Enclosures Having a Fixed Amount of Solid Material Simulated with Heterogeneous and Homogeneous Models, Intern. J. Heat and Mass Transfer, vol. 48, nos. 23-24, pp. 4748-4765, 2005.

35. M. J. S. de Lemos and R. A. Silva, Turbulent Flow Over a Layer of a Highly Permeable Medium Simulated with a Diffusion-Jump Model For The Interface, Inter. J. Heat and Mass Transfer, vol. 49, no. 3-4, pp. 546-556, 2006.

36. M. H. J. Pedras and M. J. S. de Lemos, Computation of Turbulent Flow in Porous Media using a Low-Reynolds $K$-Epsilon model and an Infinite Array of Transversally Displaced Elliptic Rods, Numer. Heat Transfer A, vol. 43, no. 6, pp. 585-602, 2003.

37. W. G. Gray and P. C. Y. Lee, On the Theorems for Local Volume Averaging of Multiphase System. Int. J. Multiphase Flow, vol. 12, pp. 401-410, 1977.

38. W. G. Gray and P. C. Y. Lee, On the Theorems for Local Volume Averaging of Multiphase System, Int. J. Multiphase Flow, vol. 3, pp. 333-340, 1977.

39. M. B. Saito and M. J. S. de Lemos, Interfacial Heat Transfer Coefficient for NonEquilibrium Convective Transport in Porous Media, Inter. Comm. Heat and Mass Transfer, vol. 32, no. 5, pp. 666-676, 2005.

40. M. B. Saito and M. J. S. de Lemos, A Correlation for Interfacial Heat Transfer Coefficient for Turbulent Flow Over an Array of Square Rods, J. Heat Transfer, vol. 128, pp. 444-452, 2006.

41. S. V. Patankar, Numerical Heat Transfer and Fluid Flow, Hemisphere, New York, 1980.

42. F. P. Incropera and D. P. DeWitt, Introduction to Heat Transfer, 3rd ed., Wiley, New York, 1996.

43. R. W. Fox and A. T. McDonald, Introduction to Fluid Mechanics, 5th ed., Wiley, New York, 1998.

44. M. Y. Chung and K. H. Luo, Unsteady Heat Transfer Analysis of an Impinging Jet, J. Heat Transfer, vol. 124, pp. 1039-1048, 2002. 